\title{
Flexible Techniques for Blind Signal Separation
}

\author{
Yongjian Zhao ${ }^{1, a}$, Manlan $\mathrm{Hao}^{2, \mathrm{~b}}$ \\ ${ }^{1}$ School of Information Engineering, Shandong University (weihai), China \\ ${ }^{2}$ Wenshang County People's Hospital, Wenshang, China \\ ajian123cn@sdu.edu.cn, bjht79@163.com
}

Keywords: Separation, Source, Component, Kurtosis, Process.

\begin{abstract}
Traditional blind source separation (BSS) approach tries to recover all source signals from observed mixtures simultaneously. However, the recovered signals suffer from an inherent ambiguity on dilation and permutation. A flexible signal extraction process is deduced on the basis of kurtosis. Correspondingly, someone can extract only a few source signals or separate all source signals in a prescribed order. Therefore, the proposed method can provide more freedom in signal separation.
\end{abstract}

\section{Introduction}

Blind source separation (BSS) is a powerful technique to recover the original sources from their mixtures without knowledge of the mixing process [1-3]. Indeed, blind is not complete since someone must use certain statistical properties of original sources. In last decades, BSS technique has been studied extensively in the domain of biomedical signal processing, speech and image processing, data mining, and so on [4,5]. The traditional BSS technique aims to recover all source signals simultaneously, but the recovered signals suffer from an inherent ambiguity on dilation and permutation. In most cases, the number of sources is large while only one or a few are desired. For example, in ECG or EEG we can obtain more than 64 sensor signals but only several signals are interested, and the rest are regarded as interfering noise. In such cases, traditional BSS technique is very computationally demanding. We start to overcome the inherent shortcomings inherent in traditional BSS methods.

According to the central limit theorem, the distribution of a sum of independent random variables tends to a Gaussian distribution [6]. Therefore, measurements of non-Gaussianity are well established and understood to recover blind source signal. As a practical measure of non-Gaussianity, kurtosis generally represents the preferred technique for blind source extraction [7-9]. In this paper, we firstly calculate the performance of kurtosis. Then a cost function is deduced on the basis of normalized kurtosis. Correspondingly, a flexible learning algorithm is derived based on the standard gradient descent rule. By the proposed method, someone can extract a desired source signal owning valuable information. Furthermore, he can separate all source signals one by one in a prescribed order, which is also important in practice. Computer simulations demonstrate its validity and usefulness.

\section{Proposed Algorithm}

The mixed sensor signals $x(k)=\left[x_{1}(k), \cdots, x_{n}(k)\right]^{T}$ is given by the following matrix equation

$$
\mathrm{x}(k)=A s(k) \text {, }
$$

where $A$ is an $\mathrm{n} \times \mathrm{n}$ unknown mixing matrix, $k$ is the discrete time index, and $s(k)=\left[s_{1}(k), \cdots, s_{n}(k)\right]^{T}$ is a vector of unknown zero-mean and unit-variant original sources. Here only the vectors $\mathrm{x}(k)$ can be observed and everything else is unknown. The original sources are assumed to be mutually independent with non-Gaussian and nonlinear autocorrelation.

In model (1), only the vectors $\mathrm{x}(k)$ can be observed and everything else is unknown. The basic problem of BSS is to estimate both the mixing matrix $A$ and the source signals $s(k)$ using observations of the mixtures $\mathrm{x}(k)$ and some statistical properties of original sources. Traditional BSS technique 
aims to recover all source signals from their mixture simultaneously [2,6]. As the mixture of independent Gaussian sources mixed by an orthogonal mixing matrix remains independent, the BSS techniques are only applicable to non-Gaussian sources, or when at most only one source is Gaussian[8,9]. Under this condition, the extraction of one original source signal is equivalent to extracting an independent component from the mixtures. Assume that someone wants to extract a desired source signal $\mathrm{y}(\mathrm{k})$, an efficient technique is to introduce an iterative process to find a weight vector w so that $y(k)=w^{T} x(k)=g^{T} s(k)$ is a good approximation to the desired source signal [4,5]. Here $g^{T}=w^{T} A$ is the global demixing vector.

Non-Gaussianity is actually of paramount importance in BSS estimation [1,4]. In recent decades, solutions based on measurements of non-Gaussianity are well established and understood for BSS problem. To use non-Gaussianity for signal separation, one must have a quantitative measure about non-Gaussianity of a random variable. As a first practical measure of non-Gaussianity, Kurtosis is the name given to the fourth-order cumulant of a random variable. Kurtosis is defined in the zero-mean case by the equation

$$
k t(y)=E\left\{y^{4}\right\}-3\left(E\left\{y^{2}\right\}\right)^{2}
$$

where $E\{\cdot\}$ denotes the statistical expectation operator.

Alternatively, the normalized kurtosis

$$
k_{4}(y)=\frac{E\left\{y^{4}\right\}}{\left[E\left\{y^{2}\right\}\right]^{2}}-3
$$

can be used. For whitened data $E\left\{x^{2}\right\}=1$, both the versions of kurtosis reduces to

$$
k t(y)=k_{4}(y)=E\left\{y^{4}\right\}-3 .
$$

Therefore, for whitened signal, the fourth moment $E\left\{x^{4}\right\}$ can be chosen instead of kurtosis for characterizing the distribution of $\mathrm{y}$. Kurtosis has many useful property. If $\mathrm{x}$ and $\mathrm{y}$ are two statistically independent random variables, one can deduce that

$$
k t(x+y)=k t(x)+k t(y) .
$$

For any scalar parameter $\delta$,

$$
k t(\delta y)=\delta^{4} k t(y) .
$$

Therefore, kurtosis is not linear with respect to its argument. Furthermore, kurtosis is the simplest statistical quantity for indicating the non-Gaussianity of a random variable. If $\mathrm{x}$ has a Gaussian distribution, its kurtosis is zero. This is the sense in which kurtosis is "normalized" when compared to the fourth moment, which is not zero for Gaussian variables. Normalized kurtosis owns the advantage that we do not need to perform the otherwise required pre-whitening and weight normalization operations. Therefore, we adopted the normalized kurtosis to represent the stochastic properties of the source signals. A cost function is formulated on the basis of normalized kurtosis as follows:

$$
\text { minimize } J(w)=-\frac{1}{4}\left|k_{4}(y)\right|
$$

subject to the constraint $\|w\|=1$

To minimize the absolute normalized value of kurtosis, we start from certain vector $w$, compute the direction in which the absolute value of the kurtosis of $y=w^{T} x$ is growing most strongly, on the basis of the available sample $x(1), \cdots, x(T)$ of mixture vector $x$, and then move the vector $w$ in that direction. Applying the standard gradient descent approach to (7), we deduce the learning rule:

$$
\begin{array}{r}
\bar{w}(t+1)=\bar{w}(t)+\mu \cdot \operatorname{sgn}\left(k_{4}(t)\right) f[y(t)] x(t) \\
w(t+1)=\bar{w}(t+1) /\|\bar{w}(t+1)\|
\end{array}
$$

where $\mu$ is the step size and the nonlinear function $f[y(t)]$ is deduced by

$$
f[y(t)]=m_{2}^{2} \frac{\partial k_{4}(y)}{\partial(y)}=y^{3}(t)-\frac{m_{4}(t)}{m_{2}(t)} y(t) .
$$


Here, $m_{p}(t)(p=2,4)$ is the $p$ th moment.

Denote that $\mathrm{s}(\mathrm{k})$ is extracted, where $\mathrm{k}$ indicates the total number of source signals being extracted so far. We exploit the knowledge of $\mathrm{s}(\mathrm{k})$ to generate the new input vector $\mathrm{x}(\mathrm{k})$ which will not include the already extracted signals. We conduct above rule by the following linear transformation:

$$
\bar{x}(k)=x(k)-w^{T} x(k)=x(k)-w^{T} A s(k) .
$$

After the deflation process shown in (11), the extracted signal can be eliminated from its mixture. The remained mixture then can undergo another separation process so as to recover the next source signal. In other words, the original sources can be recovered from their mixture one by one.

\section{Experimental Results}

We used the ABio7 dataset proposed by Barros [9], a benchmark containing a set of typical biomedical signals. Each signal had 5000 samples with zero means and unit variances. Due to space constraints, we selected three signals, denoted by s1, s2 and s3 in Fig.1. Signal s1 is an ECG and the other two are common artifacts frequently observed while measuring the ECG: electrode and respiratory artifacts. A $3 \times 3$ mixing matrix $A$ was randomly generated whose rows were $A 1=[0.79$ $-0.390 .67], A 2=\left[\begin{array}{lll}0.68 & 0.39 & 0.51\end{array}\right]$ and $A 3=\left[\begin{array}{lll}-0.51 & 0.79 & 0.77\end{array}\right]$. Three source signals were mixed with the matrix $A$. The mixed results were shown in Fig.2. We ran the proposed algorithm in this paper and the separation results were depicted in Fig.3.

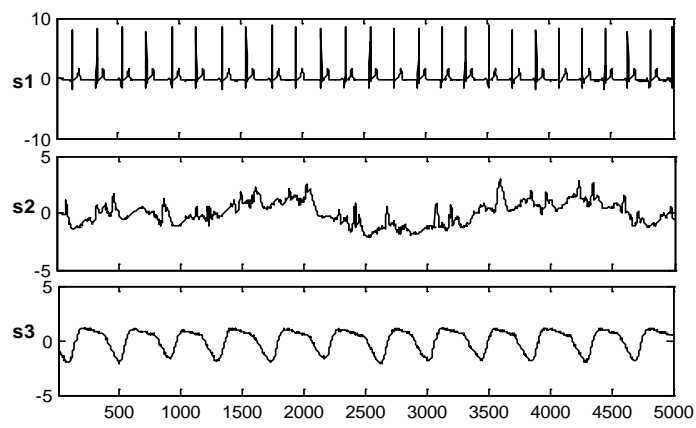

Fig.1 Original biomedical signals

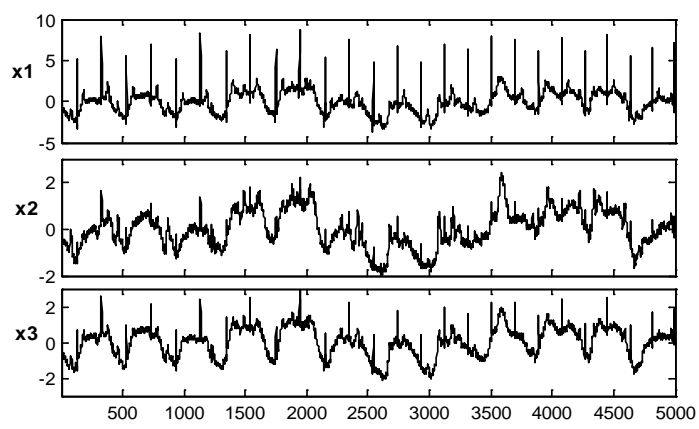

Fig.2 Signals mixed by random matrix

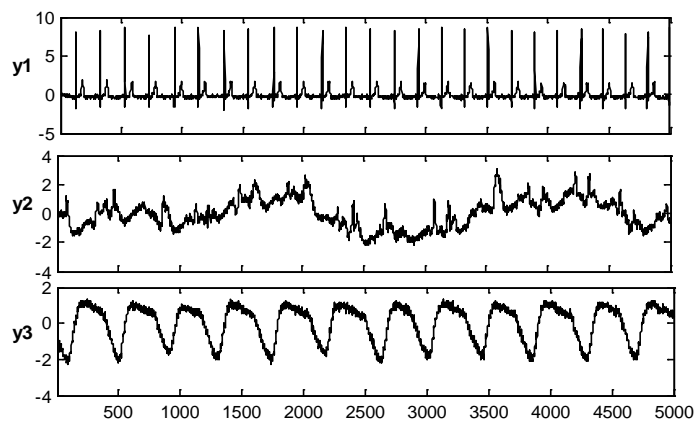

Fig.3 Signals separated by the proposed algorithm 
We also ran typical BSS methods: BSBSE in [2] and EVBSE in [5]. For simplicity, we call the proposed algorithm in this paper as NEWBSE. To further calculate the accuracy of extraction, we adopted the index of signal-to-noise ratio (SNR) in $\mathrm{dB}$ given by $\mathrm{SNR}=10 \log _{10}\left(\mathrm{~S}^{2} / \mathrm{MSE}\right)$, where $\mathrm{S}^{2}$ denotes the variance of the source and MSE denotes the mean square error between the original signal and recovered signal. Obviously, the higher SNR is, the better the performance is. To exactly compare the performance of these algorithms, we repeated these simulations 100 times independently so as to get the average and highest SNR. The simulation results are shown in Table 1 . The superiority of the proposed algorithm is obviously.

Table 1 Comparison of SNRs (dB) by three algorithms

\begin{tabular}{|r|ccl|}
\hline algorithm & BCBSE & EVBSE & NEWBSE \\
\hline average SNR & 12.5643 & 18.5637 & 21.5674 \\
highest SNR & 14.5436 & 21.6538 & 23.5639 \\
\hline
\end{tabular}

\section{Summary}

To improve signal separation performance and expand application, a flexible algorithm, called NEWBSE, has been deduced in this paper. It can efficiently separate signal mixture into their underlying components. Preliminary results on biomedical signals have confirmed its reliability. Further theoretical endeavors of the proposed method and the improved faster method are subjects for future study.

\section{Acknowledgements}

This work is supported by the National Natural Science Foundation of China (11473019), Natural Science Foundation of Shandong (ZR2014AM015).

\section{References}

[1] C. J. James and C. W. Hesse, Independent component analysis for biomedical signals, Physiological Measurements, vol.26, pp.15-39, 2005.

[2] A. K. Barros and A. Cichocki, Extraction of specific signals with temporal structure, Neural Computation, vol.13, pp.1995-2003, 2001.

[3] E. Santata, J. C. Principe and E.E. Santana, Extraction of signals with specific temporal structure using kernel methods, IEEE Transactions on Signal Processing, vol.58, no.10, pp. 5142-5150, 2010.

[4] Y. J. Zhao, B. Q. Liu and S. Wang, A robust extraction algorithm for biomedical signals from noisy mixtures, Frontiers of Computer Science in China, vol.5, no.4, pp. 387-394, 2011.

[5] Z. L. Zhang and Y. Zhang, Extraction of temporally correlated sources with its application to non-invasive fetal electrocardiogram extraction, Neurocomputing, vol.69, pp. 894-899, 2006.

[6] W. Lu and J. C. Rajapakse, ICA with reference, Neurocomputing, vol.69, pp. 2244-2257, 2006.

[7] Z. L. Zhang, Morphologically constrained ICA for extracting weak temporally correlated signals, Neurocomputing, vol.71, pp. 1669-1679, 2008.

[8] R. Llinares, J. Igual, A. Salazar and A. Camacho, Semi-blind source extraction of atrial activity by combining statistics and spectral features, Digital Signal Processing, vol.21, pp.391-403, 2011.

[9] D. De Moor (Ed.), Daisy: database for identification of systems, 1997, URL: http://www.esat.kuleuven.ac.be/sista/daisy. 